\title{
THE MULTIPLICATIVE GROUP OF ABSOLUTELY ALGEBRAIC FIELDS IN CHARACTERISTIC $p$
}

\author{
STEPHEN J. TILLMAN
}

Let $p$ be a fixed prime number, and $k$ an algebraic extension of $F=Z / p Z$. Let $F_{c}$ be the algebraic closure of $F$. It is well known that

$$
G\left(F_{c} / F\right) \approx \hat{Z} \approx \prod_{\text {all primes } q} Z_{q}
$$

where $Z_{q}$ is the $q$-adic integers. Hence $G(k / F)$ is isomorphic to a factor group of $\hat{Z}$, and is essentially described by a supernatural number,

$$
N=\prod_{\text {all primes } q} q^{r(q)}
$$

where $r(q)$ is either finite or infinite, and $q^{r(q)}$ is the degree of the maximal $q$-extension of $F$ in $k$.

We shall show that the multiplicative group of $k, k^{*}$, is isomorphic to a subgroup of

$$
Q / Z \approx \sum_{\text {all primes } q} Z\left(q^{\infty}\right),
$$

where $Z\left(q^{\infty}\right)$ is the $q$-primary part of $Q / Z$. Thus $k^{*}$ is also described by a supernatural number,

$$
M=\prod_{\text {sll primes } q} q^{*(q)},
$$

where $s(q)$ is either finite or infinite and $q^{s(q)}$ is the "order" of the $q$-Sylow subgroup of $k^{*}$.

We shall see that the $s(q)$ 's are completely determined by the $r(q)$ 's defined in (1). In particular we shall easily be able to see when $k^{*}$ is $q$-divisible for any prime $q$.

Definition. For any group $G$, we say $G$ has condition $T$ if $\forall a, b \in G, \exists$ a cyclic subgroup $H$ of $G$ such that $a, b \in H$.

Proposition. Any group with condition $T$ is isomorphic to a subgroup of either $Q$ or $Q / Z$.

Proof (The proof of this proposition is dUE to Professor Michael I. Rosen). Suppose $G$ is a group with condition $T$. Then $G$

Received by the editors July 11, 1968 . 
is either a torsion group, or a torsion free group, for if $a, b \in G$, such that $a$ has finite order and $b$ has infinite order, then there exists no cyclic subgroup containing both $a$ and $b$, contradicting the assumption that $G$ has condition $T$. We shall assume that $G$ is a torsion group, and prove the proposition only in this case. $G$ is an abelian torsion group, hence it is isomorphic to the direct sum of its primary parts $G_{q}$. We will show that $G_{q}$ is either cyclic or isomorphic to $Z\left(q^{\infty}\right)$. This is sufficient to prove our assertion since $Z\left(q^{\infty}\right)$ is the $q$-primary component of $Q / Z$. Obviously if $G_{q}$ is finite it must be cyclic. Suppose $G_{q}$ is infinite, and let $h_{1}, h_{2}, h_{3}, \cdots$ be a sequence of distinct elements of $G_{q}$. Let $H_{r}$ be the subgroup generated by $h_{1}, \cdots, h_{r} . H_{r}$ is a finite cyclic group. Consider the chain $H_{1} \subseteq H_{2} \subseteq H_{3} \subseteq \cdots$. This chain cannot break off. Let $H=\bigcup_{i=0}^{\infty} H_{i}$. It is easily seen that $H$ is isomorphic to $Z\left(q^{\infty}\right)$. Since $Z\left(q^{\infty}\right)$ is a divisible group, $H$ is a direct summand of $G_{q}$. If it were a proper direct summand, $G_{q}$ would contain a subgroup isomorphic to $Z / q Z \oplus Z / q Z$. This is impossible since $Z / q Z \oplus Z / q Z$ does not have condition $T$, and condition $T$ is inherited by subgroups. Thus $H=G_{q}$ and $G_{q} \approx Z\left(q^{\infty}\right)$. QED

REMARK. $k^{*}$ has condition $T$, and is torsion; hence by the above proposition we can write

$$
k^{*} \approx \sum_{\text {all primes } q} G_{q}
$$

where $G_{q} \subseteq Z\left(q^{\infty}\right)$. Note that for any prime $q, k^{*}$ is $q$-divisible if and only if $G_{q}$ is isomorphic to either (0) or $Z\left(q^{\infty}\right)$.

Proposition 1. $G_{p} \approx(0)$.

Proof. Suppose not. Then $k^{*}$ contains a nontrivial element of order $p$. This element would satisfy $f(x)=x^{p}-1=0$ over $k[x]$; but since the characteristic at $k$ is $p, 1$ is the only solution to $f(x)=0$.

QED

Definition. For any prime $q \neq p$ define $l(q)$ as the smallest positive integer such that $p^{l(q)} \equiv 1(q)$.

Proposition 2. For any prime $q \neq p$, if $l(q) \nmid N$, then $G_{q} \approx(0)$.

Proof. If $G_{q} *(0)$, then some finite subfield, $E$, of $k$ has a nontrivial $q$-Sylow subgroup of its multiplicative group. We shall show that this is impossible. Let $E$ be any finite subfield of $k$. Then for some $n \in Z$, $E^{*}$ has $p^{n}-1$ elements, and $l(q) \nmid n$, for if it did, it would certainly divide $N$. Hence $n=u l(q)+v, 0<v<l(q) . \quad p^{n}-1=p^{v}\left(p^{u l(q)}-1\right)$ $+\left(p^{v}-1\right)$. But by the definition of $l(q), p^{u l(q)} \equiv 1(q)$, and $p^{v} \neq \equiv 1(q)$, hence $p^{n}-1 \equiv p^{v}-1 \neq 0(q)$, so $q \nmid p^{n}-1$, and $E^{*}$ has no nonzero $q$-subgroup. QED 
REMARK. Notice that $F$ has an $l(q)$-extension in $k$ if and only if $l(q) \mid N$.

Proposition 3. Let $q \neq p$ be any prime, and assume $F$ has an $l(q)$ extension in $k$. Then $k^{*}$ is q-divisible if and only if the maximal q-extension of $F$ in $k$ is infinite i.e., $r(q)=\infty$.

Proof. Suppose the maximal $q$-extension of $F$ in $k$ is infinite. Since we are assuming $F$ has an $l(q)$-extension in $k, \exists$ a finite subfield $E$ such that $E^{*}$ has $p^{n}-1$ elements and $n=l(q) m$. We have

$$
p^{n}-1=\left(p^{l(q)}-1\right)\left(p^{l(q)(m-1)}+p^{l(q)(m-2)}+\cdots+p^{l(q)}+1\right) .
$$

But by definition $p^{l(q)} \equiv 1(q)$ hence $q \mid p^{n}-1$, so $G_{q} \neq(0)$ (defined in equation (3)). Thus we must show $G_{q} \approx Z\left(q^{\infty}\right)$. Since we are assuming $r(q)=\infty$, it suffices to show that $q$-extensions have strictly increasing $q$-Sylow subgroups of their multiplicative groups. We must show that if

$$
p^{l(q) q^{n}}-1=q^{\alpha(n)} t_{n}, \quad q \nmid t_{n},
$$

then $\alpha(n)>\alpha(n-1)$. (Equation (4) defines $\alpha(n)$.)

$$
\begin{aligned}
p^{l(q) q^{n}}-1 & =\left(p^{l(q) q^{n-1}}-1\right)\left(p^{l(q) q^{n-1}(q-1)}+p^{l(q) q^{n-1}(q-2)}+\cdots+1\right) \\
& =q^{\alpha(n-1)} t_{n-1}\left(p^{l(q) q^{n-1}(q-1)}+\cdots+1\right),
\end{aligned}
$$

so $\alpha(n)>\alpha(n-1)$ because $\left(p^{l(q) q^{n-1}(q-1)}+\cdots+1\right)$ has $q$ terms, each congruent to $1 \bmod q$, hence is divisible by $q$.

Now suppose the maximal $q$-extension of $F$ in $k$ is finite. We saw in the proof of the first part of this proposition that the assumption $l(q) \mid N$ implies $G_{q} \neq(0)$. Hence if $k^{*}$ were $q$-divisible, we could find a finite subfield $E$ of $k$ such that the $q$-Sylow subgroup of $E^{*}$. has more than $q^{\alpha(r(q))}$ elements. Suppose $E$ is any such finite subfield. There is no loss of generality in assuming that $E$ contains an $l(q)$-extension and a maximal $q$-extension of $F$ in $k$. Thus $E^{*}$ has $p^{l(q) q^{r(q)} m}-1$ elements, where $q \nmid m$. But

$$
\begin{aligned}
p^{l(q) q^{r(q) m}}-1 & =\left(p^{l(q) q^{r(q)}}-1\right)\left(p^{l(q) q^{r(q)(m-1)}}+\cdots+1\right) \\
& =q^{\alpha(r(q))} t_{r(q)}\left(p^{l(q) q^{r(q)(m-1)}}+\cdots+1\right),
\end{aligned}
$$

and since $q \nmid m$ and $p^{l(q)} \equiv 1(q), q \nmid\left(p^{l(q) q^{r(q)}(m-1)}+\cdots+1\right)$; hence the $q$-Sylow subgroup of $E^{*}$ has only $q^{\alpha(r(q))}$ elements, so we have a contradiction. Thus $k^{*}$ is not $q$-divisible. QED

The following lemma is due to Van der Waerden (see [1, p. 58]).

LEMMA. Let $a, r$ be integers $>0$ and $a$ an integer $>1$. Let $q$ be a prime number and 


$$
T=\left(a^{q^{r}}-1\right) /\left(a^{q^{r-1}}-1\right) .
$$

If a prime $p$ divides $T$ and $a^{q^{-1}}-1$, then $q=p$. If $q$ divides $T$, then $q$ divides $a^{q^{-1}}-1$. Finally, if $q>2$ or $r>1$, then $T \not \equiv 0\left(q^{2}\right)$.

Proof. We have

$$
T=\left(a^{q^{r-1}}-1\right)^{q-1}+q\left(a^{q^{r-1}}-1\right)^{q-2}+\cdots+q .
$$

This proves all the statements except when $q=2$, and in that case, $T=\left(a^{2-1}-1\right)+2$, so that these assertions are also obvious. QED

Definition. We define the function $\beta(q)$ for all primes $q \neq p$ as follows:

$$
\begin{aligned}
p^{l(q)}-1 & =q^{\beta(q)} t_{q}, & & q \nmid t_{q}, q \neq 2, \\
p^{2}-1 & =2^{\beta(2)} t_{2}, & & t_{2} \text { odd }, q=2 .
\end{aligned}
$$

Proposition 4. Let $q$ be a prime such that $G_{q}$ is finite and nontrivial. Then

$$
\begin{array}{ll}
s(q)=\beta(q)+r(q) & \text { if } q \neq 2, \\
s(2)=\beta(2)+r(2)-1 & \text { if } q=2, p \neq 2,
\end{array}
$$

where $r$ and $s$ are defined in equations (1) and (2) respectively.

Proof. Recall that the order of $G_{q}$ is $q^{s(q)}$. We are assuming $G_{q}$ is finite and nontrivial, so $F$ has an $l(q)$-extension in $k$, and its maximal $q$-extension in $k$ is finite. We also saw in the proof of Proposition 3 that the order of $G_{q}$ is $q^{\alpha(r(q))}$, where $\alpha$ is defined in equation (4). Then by looking at the definitions of $\alpha$ and $\beta$, and by successively applying the lemma, where we let $a=p^{l(q)}$, we see that (recalling $l(2)=1$ )

$$
\begin{aligned}
& \alpha(r(q))=\beta(q)+r(q), \quad q \neq 2, \\
& \alpha(r(2))=\beta(2)+r(2)-1 . \quad \text { QED }
\end{aligned}
$$

Theorem. $G(k / F)$ completely determines $k^{*}$ in the sense of the supernatural number $N$ explicitly determining the supernatural number $M$.

Proof. Given any $q$, if $q=p, G_{p} \approx(0)$ by Proposition 1 , so $s(p)=0$. If $l(q) \nmid N, G_{q} \approx(0)$ by Proposition 2, so $s(q)=0$. If $l(q) \mid N$ and $r(q)$ $=\infty, G_{q} \approx Z\left(q^{\infty}\right)$ by Proposition 3 , so $s(q)=\infty$. If $l(q) \mid N$ and $r(q)<\infty$, $G_{q}$ is finite, nontrivial and, by Proposition $4, s(q)=\beta(q)+r(q)$ if $q \neq 2, s(2)=\beta(2)+r(2)-1$. QED

\section{BIBLIOGRAPHY}

1. Serge Lang, Algebraic numbers, Addison-Wesley, Reading, Mass., 1964.

BROWN UNIVERSITY 